\title{
Excitation Mechanisms and Characterization of a Multi-Ionic Xenon Laser
}

\author{
H. Sobral, M. Raineri, D. Schinca, M. Gallardo, and R. Duchowicz
}

\begin{abstract}
The emission characteristics of an ultraviolet-visible pulsed multi-ionic xenon laser were studied through time-resolved spectroscopy and the results were interpreted using a collisionalradiative theoretical model. This analysis includes more than 20 laser lines belonging to several ionic species (Xe III-VIII). Depending on the experimental conditions, different temporal distributions of the laser lines and their corresponding spontaneous emissions can be observed. In particular, laser emission presents temporal oscillations near threshold. Pumping processes for the laser transitions have been analyzed by using this model. Relativistic Hartree-Fock calculations of laser level lifetimes and radiative transition probabilities were performed. Experimental laser gain for several transitions were obtained and compared with the theoretical values derived from the calculations.
\end{abstract}

Index Terms-Collisional-radiative model, gas discharge, gas lasers, xenon laser.

\section{INTRODUCTION}

$\mathbf{S}$ NCE THE advent of the laser, capillary discharges have been used to produce laser action in the ultraviolet (UV), visible, and infrared (IR) range [1]-[4]. In particular, lowpressure xenon plasma excited by pulsed high-current-highvoltage electrical discharges produces high-gain laser transitions in the near UV and visible range. This laser output has been used for pumping dye lasers [5]-[7] and for studying injection-locking phenomena in CW dye lasers [8], [9].

Both from a basic and practical point of view, it is important to know which are the levels and ionic species involved in laser emission. Duchowicz et al. [10] showed that most visible laser emissions belong to $\mathrm{Xe} \mathrm{V}$ while UV emissions belong in general to Xe VII. In recent works, most of the unresolved laser lines were attributed to Xe V [11], Xe VII [12], and Xe VIII [13].

Manuscript received December 9, 1998; revised June 7, 1999. This work was supported by the Consejo Nacional de Investigaciones Cientificas y Técnicas (CONICET), by the Comisión de Investigaciones Cientificas de la Provincia de Buenos Aires (CICBA), and by the Facultad de Ciencias Exactas, Universidad Nacional de La Plata.

H. Sobral and D. Schinca are with Centro de Investigaciones Opticas, 1900 La Plata, Argentina. They are also with CICBA, 1900 La Plata, Argentina, and with the Physics Department, Universidad Nacional de La Plata, $1900 \mathrm{La}$ Plata, Argentina.

M. Raineri is with Centro de Investigaciones Opticas, 1900 La Plata, Argentina. He is also with CICBA, 1900 La Plata, Argentina.

M. Gallardo is with the Centro de Investigaciones Opticas, 1900 La Plata, Argentina. He is also with CONICET, 1916 Buenos Aires, Argentina.

R. Duchowicz is with the Centro de Investigaciones Opticas, 1900 La Plata, Argentina. He is also with CONICET, 1916 Buenos Aires, Argentina, and with the Physics Department, Universidad Nacional de La Plata, 1900 La Plata, Argentina.

Publisher Item Identifier S 0018-9197(99)06785-8.
Following another line of research, Papayoanou et al. [14] reported on the parametrization of a xenon plasma laser including observations about the temporal distribution of the output. Sasaki and Saito [15] have obtained double-pulsed output when the plasma was excited with a long current pulse. In a previous work [16], we presented experimental results about the temporal evolution of visible laser lines together with a simple model to simulate this behavior. After that, we performed an extensive study on the temporal characteristics of the spontaneous emission of a low-density pulsed discharge plasma. The results were interpreted through a collisionalradiative theoretical model [17].

This paper extends the previous temporal study of the laser emission to more than 20 lines in the UV-visible region. Based on our previous plasma model that considers the population behavior of Xe I-IX species [17], we have now also included stimulated emission terms to take into account different laser transitions. On the basis of this model, we analyze the population mechanisms responsible for most of the classified laser xenon transitions corresponding to $\mathrm{Xe}$ III, $\mathrm{Xe} \mathrm{V}, \mathrm{Xe}$ VII, and Xe VIII, in this region. We also performed laser gain analyses for several lines by using level parameters (lifetimes and radiative transition rates) obtained from relativistic Hartree-Fock calculations; these results are compared with the corresponding experimental values.

\section{EXPERIMENTAL SETUP AND RESUlTS}

The experimental setup is similar to that used in [16]. The discharge tube was $1.3 \mathrm{~m}$ long and has a $5-\mathrm{mm}$ bore, with cold electrodes in side arms at the ends. Excitation was achieved using a capacitor bank having an overall capacitance ranging from 100 to $420 \mathrm{nF}$ and charged to a voltage up to $18 \mathrm{kV}$, which yields peak current pulses of about $3 \mathrm{kA}$ having a temporal width between 1-3 $\mu$ s full-width at half-maximum (FWHM). Xenon pressure was varied between 5-100 mtorr. The resonant cavity consisted of two multilayer coated spherical mirrors of $1.5-\mathrm{m}$ radius of curvature located in a nearly confocal configuration. The light emitted longitudinally was focused into a $0.5-\mathrm{m}$ focal length scanning monochromator and detected with a UV-visible photomultiplier. Its output was amplified and displayed by a $200-\mathrm{MHz}$ digital oscilloscope and recorded by a plotter.

The temporal study carried out previously in the visible region was extended into the UV region as far as $2000 \AA$, studying more than 20 transitions corresponding to different ionic degrees (Xe III-VIII). 


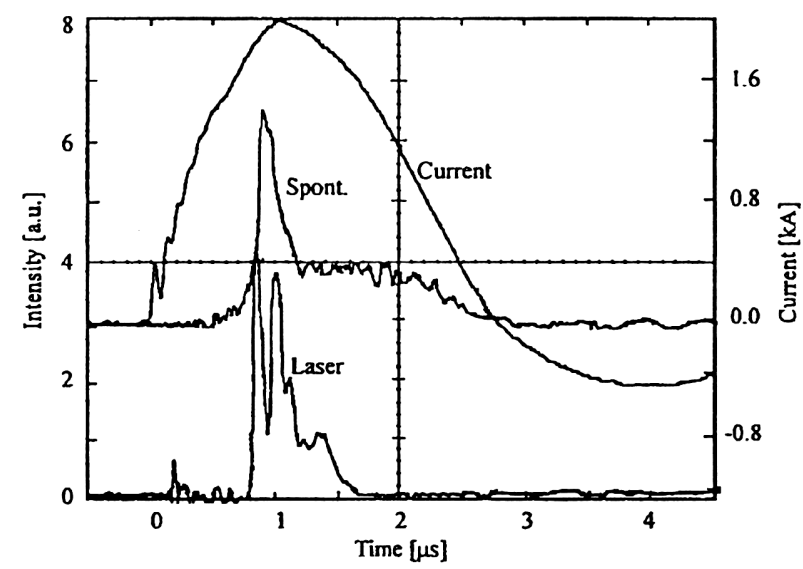

Fig. 1. Oscillation behavior near laser threshold for the $\lambda \equiv 3350.03 \AA$ transition belonging to Xe VIII; pressure: $12 \mathrm{mtorr}$.

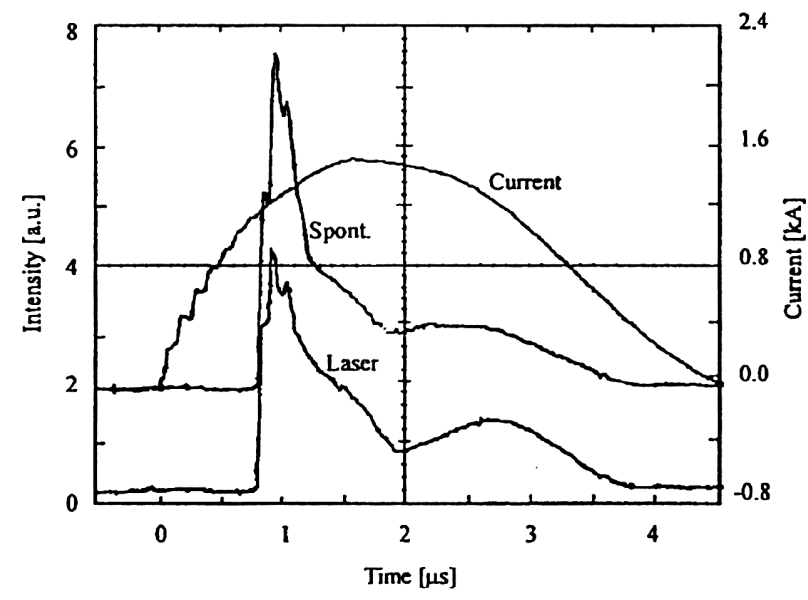

Fig. 2. Temporal distribution for the $\lambda \equiv 3645.48-\AA$ line (Xe VII); pressure: 10 mtorr.

Depending on the mirror reflectivity and experimental conditions, several ions can lase simultaneously. As the stage of ionization increases, the onset of lasing is delayed further with respect to the beginning of the current pulse. These results were also observed in previous studies of the spontaneous emission [10], [17].

We have analyzed the temporal distribution of the laser and the corresponding spontaneous emission of each line studied under different experimental conditions. In these situations, we removed the output mirror to compare the former (that depends on both the upper and lower laser level population) with the latter (proportional to the upper level population only). Near threshold, laser emission oscillations occurs in most of the observed lines, especially for high ionic degrees. Fig. 1 shows the temporal distribution of the laser emission near threshold for a line belonging to Xe VIII $(\lambda=3350.03 \AA)$ together with the corresponding spontaneous emission output and the current pulse for reference. During the temporal region around the spontaneous peak, the laser emission exhibits oscillations. After that, the ion population and the spontaneous and laser emission decay.

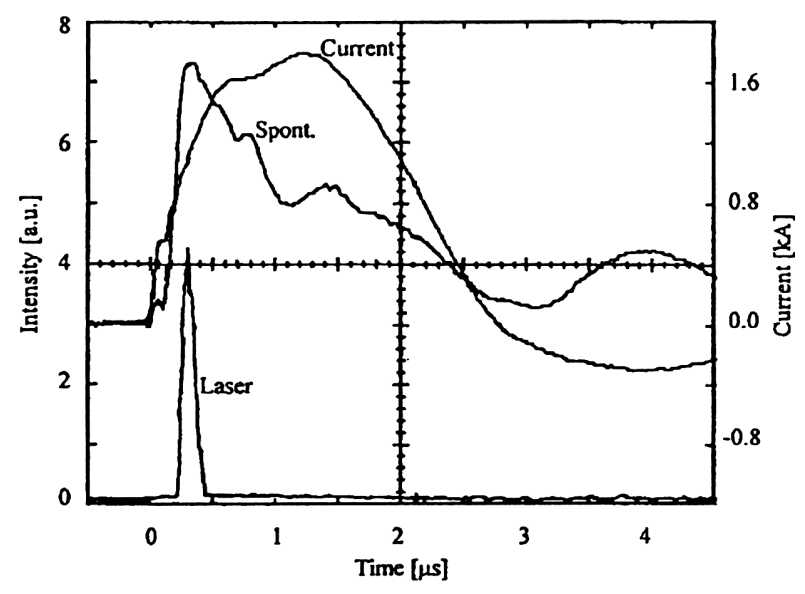

Fig. 3. Spontaneous and laser emission well above threshold for the $\lambda=4060.45-\AA$ line $(\mathrm{Xe} \mathrm{III)}$; pressure: $15 \mathrm{mtorr}$.

When pumping is increased either by increasing the discharge voltage or by decreasing the pressure, oscillations tend to disappear and laser emission follows the temporal shape of the spontaneous emission. This behavior is shown in Fig. 2 for the 3645.48- $\AA$ line (Xe VII), where laser and spontaneous temporal distributions are similar. This particular line presents, when pumping is above threshold, a flat temporal profile during almost all of the current pulse.

Well above laser threshold, the output consists of a single pulse appearing at the beginning of the corresponding spontaneous pulse. The large initial gain obtained under these conditions produces a depopulation of the upper laser level which usually cannot recover to generate a positive population inversion. Fig. 3 shows this behavior for the 4060.45- $\AA$ line, belonging to Xe III.

\section{MODEL}

The model used to analyze the temporal evolution of the population densities is similar to that used in [17]. If $N_{j}^{z}$ is the population density of the $j$ th level of ion $z$ in a plasma with no cavity effects, the set of equations that describe its temporal evolution is the following:

$$
\begin{aligned}
\frac{d N_{j}^{z}}{d t S_{p .} .} & n_{e}(t) \sum_{i<j} R_{i j}^{z} N_{i}^{z}-n_{e}(t) \sum_{k>j} R_{j k}^{z} N_{j}^{z} \\
& -\sum_{i<j}\left(A_{j i}^{z}+n_{e}(t) D_{j i}^{z}\right) N_{j}^{z} \\
& +\sum_{k>j}\left(A_{k j}^{z}+n_{e}(t) D_{k j}^{z}\right) N_{k}^{z}-n_{e}(t) \sum_{i} S_{j i}^{z} N_{j}^{z} \\
& +n_{e}(t) \sum_{k} S_{k j}^{z-1} N_{k}^{z-1}+n_{e}(t) \kappa_{d_{0 j}}^{z} N_{0}^{z+1} \\
& -n_{e}(t) \delta_{j 1} \sum_{i} \kappa_{d_{j i}-1}^{z-1} N_{j}^{z}
\end{aligned}
$$

where the electron density $n_{e}$ can be calculated from the charge balance equation, $R_{i j}^{z}$ is the excitation rate coefficient from level $i$ to level $j$ of the ion $z$ (corresponding to the $z-1$ times ionized xenon), $D_{j i}^{z}$ is the deexcitation rate coefficient, $A_{j i}^{z}$ is the radiative transition rate, $S_{j i}^{z}$ is the electron-impact 
ionization rate from a $j$ th level of the ion $z$ to the level $i$ of the ion $(z+1)$, and $\kappa_{d_{k j}}^{z}$ is the dielectronic recombination coefficient from the $k$ th level of the ion $z+1$ to the level $j$ of the ion $z$. The excitation and ionization rates were calculated using the semi-empirical formulas of Van Regemorter and Lotz, respectively, cited in [18]; $D_{j i}^{z}$ is calculated through its relation with the excitation rate [18] $g_{i} R_{i j}^{z} \equiv g_{j} D_{j i}^{z} \exp \left(-\Delta E / T_{e}\right)$, where $g_{i}$ and $g_{j}$ are the statistical weights of levels $i$ and $j$, and $T_{e}$ is the electron temperature. Since our plasma has a moderate electron density [17], the two-body recombination process (dielectronic and radiative recombination) prevails. Both processes can be calculated by Sobelman [18, pp. 118 and 122] and show that dielectronic recombination is the dominant term. We will restrict the number of ions to $z \leq 9$ since no experimental evidence of the existence of $\mathrm{Xe} \mathrm{X}$ was found under our conditions. The system must also satisfy the initial condition that $N_{1}^{1}$ takes on a value of about $10^{15}$ $\mathrm{cm}^{-3}$ (depending on the filling pressure), while the rest of populations are zero.

When the cavity is taken into account, the temporal evolution of the levels that give rise to laser action are also affected by the stimulated emission terms. If $N_{u}^{z}$ and $N_{l}^{z}$ represent the population densities for the upper and lower levels corresponding to each laser line, and $n^{z}$ is the corresponding total number of photons inside the cavity, their temporal evolution must satisfy the following set of equations [19]:

$$
\begin{aligned}
{\frac{d N_{u}^{z}}{d t} S t .}_{S t}^{z} & =\frac{d N_{u}^{z}}{d t} S p . \\
{\frac{d N_{l}^{z}}{d t} S t .}_{S t} & ={\frac{d N_{l}^{z}}{d t} S p .}_{S n^{z}\left[N_{l}^{z}-N_{u}^{z}\right]}+K n^{z}\left[N_{u}^{z}-N_{l}^{z}\right] \\
\frac{d n^{z}}{d t} & =V_{c} K n^{z}\left[N_{u}^{z}-N_{l}^{z}\right]+V_{c} K N_{u}^{z}-\gamma_{c} n^{z}
\end{aligned}
$$

where $\gamma_{c}$ is the inverse lifetime of the photons in the cavity, $V_{c}$ is the cavity volume, and $K$ is the coupling constant given by

$$
K=A_{u l}^{z}\left(4 \pi^{2} V_{c} \frac{1}{\lambda^{3}} \frac{\Delta \lambda}{\lambda}\right)^{-1}
$$

where $\lambda$ is the wavelength of the transition and $\Delta \lambda / \lambda$ is its relative linewidth.

\section{SPECTROSCOPIC PARAMETERS}

To analyze the excitation processes that give rise to stimulated emission, the lifetime and transition probability for the main laser transitions in the UV-visible region were calculated. For this purpose, theoretical determinations from relativistic Hartree-Fock (RHF) $a b$ initio calculations and energy matrix diagonalization [20] were used. In order to obtain the best values for lifetimes and spontaneous transition probabilities, semi-empirical calculations using energy parameters adjusted from least-squares calculations were performed. Rydberg series interactions were also included for a better fit of the theoretical values of the experimental energy level values.

Under our experimental conditions, we did not observe any Xe II laser lines. For Xe III, RHF calculations were carried out using energy parameters from Persson et al. [21]. Although Xe IV laser lines do exist, they appear with weak intensity
TABLE I

\begin{tabular}{|c|c|c|c|c|c|}
\hline $\begin{array}{c}\lambda[\AA]^{(a)} \\
(\text { air })\end{array}$ & Ion & $\begin{array}{c}\text { Classification } \\
\qquad E_{3} \rightarrow E_{2}\end{array}$ & $\begin{array}{c}\tau_{3} \\
{[\mathrm{~ns}]}\end{array}$ & $\begin{array}{c}\tau_{2} \\
{[\mathrm{~ns}]}\end{array}$ & $\begin{array}{c}\mathrm{A}_{32} \\
{\left[10^{7} \mathrm{~s}^{-1}\right]}\end{array}$ \\
\hline 2315.43 & VII & $5 \mathrm{~s} 4 \mathrm{f}\left({ }^{2} S\right)^{1} \mathrm{~F}_{3} \rightarrow 5 \mathrm{p}^{2}\left({ }^{1} D\right)^{1} \mathrm{D}_{2}$ & 20.4 & 0.38 & 6.0 \\
\hline 3246.99 & VIII & $6 \mathrm{~d}\left({ }^{1} S\right)^{2} D_{5 / 2} \rightarrow 5 \mathrm{f}\left({ }^{1} S\right)^{2} \mathrm{~F}_{7 / 2}$ & 3.3 & 0.37 & 0.2 \\
\hline 3305.96 & $\mathrm{~V}$ & $5 \mathrm{~s}^{2} 5 \mathrm{p} 4 \mathrm{f}\left({ }^{2} P\right)^{3} \mathrm{G}_{3} \rightarrow 5 \mathrm{~s}^{2} 5 \mathrm{p} 5 \mathrm{~d}\left({ }^{2} P\right)^{3} \mathrm{~F}_{2}$ & 9.9 & 0.78 & 3.0 \\
\hline 3330.84 & $\mathrm{~V}$ & $5 \mathrm{~s}^{2} 5 \mathrm{p} 4 \mathrm{f}\left({ }^{2} P\right)^{3} \mathrm{~F}_{4} \rightarrow 5 \mathrm{~s}^{2} 5 \mathrm{p} 5 \mathrm{~d}\left({ }^{2} P\right)^{3} \mathrm{~F}_{3}$ & 12.0 & 0.71 & 4.8 \\
\hline 3350.03 & VIII & $6 \mathrm{~d}\left({ }^{1} S\right)^{2} D_{3 / 2} \Rightarrow 5 \mathrm{f}\left({ }^{1} S\right)^{2} \mathrm{~F}_{5 / 2}$ & 3.1 & 0.50 & 0.1 \\
\hline 3645.48 & VII & $5 \mathrm{~s} 4 \mathrm{f}\left({ }^{1} S\right)^{1} \mathrm{~F}_{3} \rightarrow 5 \mathrm{p}^{2}\left({ }^{3} P\right)^{3} \mathrm{P}_{2}$ & 20.4 & 0.12 & 0.5 \\
\hline 3803.26 & $\mathrm{~V}$ & $5 \mathrm{~s}^{2} 5 \mathrm{p} 4 \mathrm{f}\left({ }^{2} P\right)^{3} \mathrm{D}_{1} \rightarrow 5 \mathrm{~s}^{2} 5 \mathrm{p} 5 \mathrm{~d}\left({ }^{2} P\right)^{3} \mathrm{P}_{0}$ & 8.3 & 0.04 & 1.7 \\
\hline 3973.01 & $\mathrm{~V}$ & $5 \mathrm{~s}^{2} 5 \mathrm{p} 4 \mathrm{f}\left({ }^{2} P\right)^{3} \mathrm{D}_{1} \rightarrow 5 \mathrm{~s}^{2} 5 \mathrm{p} 5 \mathrm{~d}\left({ }^{2} P\right)^{3} \mathrm{P}_{1}$ & 8.3 & 0.04 & 1.4 \\
\hline 4060.45 & III & $5 s^{2} 5 p^{3}\left({ }^{2} p\right) 6 p^{1} D_{2} \rightarrow 5 s^{2} 5 p^{3}\left({ }^{2} p\right) 6 s^{1} P_{1}$ & 3.1 & 0.10 & 10.0 \\
\hline 4305.69 & $\mathrm{~V}$ & $5 \mathrm{~s}^{2} 5 \mathrm{p} 4 \mathrm{f}\left({ }^{2} P\right)^{3} \mathrm{D}_{2} \rightarrow 5 \mathrm{~s}^{2} 5 \mathrm{p} 5 \mathrm{~d}\left({ }^{2} P\right)^{3} \mathrm{P}_{1}$ & 8.6 & 0.04 & 1.2 \\
\hline 4558.49 & $\mathrm{~V}$ & $5 \mathrm{~s}^{2} 5 \mathrm{p} 4 \mathrm{f}\left({ }^{2} P\right)^{3} \mathrm{~F}_{2} \rightarrow 5 \mathrm{~s} 5 \mathrm{p}^{3}\left({ }^{2} P\right)^{2} \mathrm{P}_{1}$ & 8.8 & 0.07 & 0.5 \\
\hline 4954.13 & $\mathrm{~V}$ & $5 \mathrm{~s}^{2} 5 \mathrm{p} 4 \mathrm{f}\left({ }^{2} P\right)^{1} \mathrm{G}_{1} \rightarrow 5 \mathrm{~s}^{2} 5 \mathrm{p} 5 \mathrm{~d}\left({ }^{2} P\right)^{1} \mathrm{~F}_{3}$ & 38.3 & 0.04 & 1.8 \\
\hline 5007.80 & $\mathrm{~V}$ & $5 \mathrm{~s}^{2} 5 \mathrm{p} 4 \mathrm{f}\left({ }^{2} P\right)^{3} \mathrm{D}_{3} \rightarrow 5 \mathrm{~s}^{2} 5 \mathrm{p} 5 \mathrm{~d}\left({ }^{2} P\right)^{i} \mathrm{D}_{2}$ & 9.2 & 0.05 & 1.0 \\
\hline 5159.08 & $\mathrm{~V}$ & $5 \mathrm{~s}^{2} 5 \mathrm{p} 4 \mathrm{f}\left({ }^{2} P\right)^{3} \mathrm{~F}_{4} \rightarrow 5 \mathrm{~s}^{2} 5 \mathrm{p} 5 \mathrm{~d}\left({ }^{2} P\right)^{3} \mathrm{D}_{3}$ & 10.4 & 0.04 & 1.0 \\
\hline 5260.19 & $\mathrm{~V}$ & $5 s^{2} 5 p 4 f\left({ }^{2} P\right)^{!} F_{3} \rightarrow 5 s^{2} 5 p 5 d\left({ }^{2} P\right)^{3} D_{2}$ & 9.5 & 0.05 & 0.8 \\
\hline 5352.92 & $\mathrm{~V}$ & $5 \mathrm{~s}^{2} 5 \mathrm{p} 4 \mathrm{f}\left({ }^{2} P\right)^{3} \mathrm{~F}_{3} \rightarrow 5 \mathrm{~s}^{2} 5 \mathrm{p} 5 \mathrm{~d}\left({ }^{2} P\right)^{3} \mathrm{P}_{2}$ & 9.7 & 0.04 & 0.9 \\
\hline 5394.62 & $\mathrm{~V}$ & $5 \mathrm{~s}^{2} 5 \mathrm{p} 4 \mathrm{f}\left({ }^{2} P\right)^{3} \mathrm{~F}_{2} \rightarrow 5 \mathrm{~s}^{2} 5 \mathrm{p} 5 \mathrm{~d}\left({ }^{2} P\right)^{3} \mathrm{D}_{1}$ & 8.8 & 0.06 & 0.6 \\
\hline 5955.67 & $\mathrm{~V}$ & $5 \mathrm{~s}^{2} 5 \mathrm{p} 4 \mathrm{f}\left({ }^{2} P\right)^{\prime} \mathrm{D}_{2} \rightarrow 5 \mathrm{~s}^{2} 5 \mathrm{p} 5 \mathrm{~d}\left({ }^{2} P\right)^{\prime} \mathrm{P}_{1}$ & 8.2 & 0.07 & 0.5 \\
\hline
\end{tabular}

MaIn Classified Ionic Xenon laser Transitions IN the UV-VISIBLE REgION: THEORETICAL UPPER LIFETIME $\tau_{3}$, LOWER Lifetime $\tau_{2}$, and Spontaneous Transition Probability $A_{32}$

(a) Wavelengths were taken from [24].

[22] and seem to be of little practical interest. Despite this fact, the model does take into account the dynamics of these transitions. For Xe V, similar calculations were performed with parameters taken from [11] for the even parity levels and from [23] for the odd parity levels. Spectroscopic results do not show any laser lines belonging to Xe VI. For Xe VII, almost all the experimental energy levels of the $5 \mathrm{~s} 4 \mathrm{f}$ configuration are unknown. In such a case, it is not possible to optimize the parameters using the previously mentioned technique; therefore, $a b$ initio calculations were carried out. Since Xe VIII has a single electron out of a closed shell, inclusion of the Rydberg series becomes important [20]. $A b$ initio calculations were also used in this case including the $n s$ and $n p$ series up to $n=15$ and $n d$ and $n f$ series to $n=12$.

Table I shows the results obtained for the main classified transitions in these ions. In general, the spontaneous transition probability is about $10^{7}-10^{8} \mathrm{~s}^{-1}$, and the lifetime of the upper level lies between 3-40 ns, while the lifetime of the lower level is one or two orders of magnitude lower.

\section{PUMPING Processes}

According to [17], we assume that the main excitation and deexcitation processes are radiative and collisional. In order to understand the population inversion mechanisms, the set of equations (1) was solved for typical discharge conditions. RHF $a b$ initio calculations were carried out for each one of the ions. They included all the relevant configurations that show interaction with those to which the laser levels belong. From these results, the levels that showed larger oscillator 


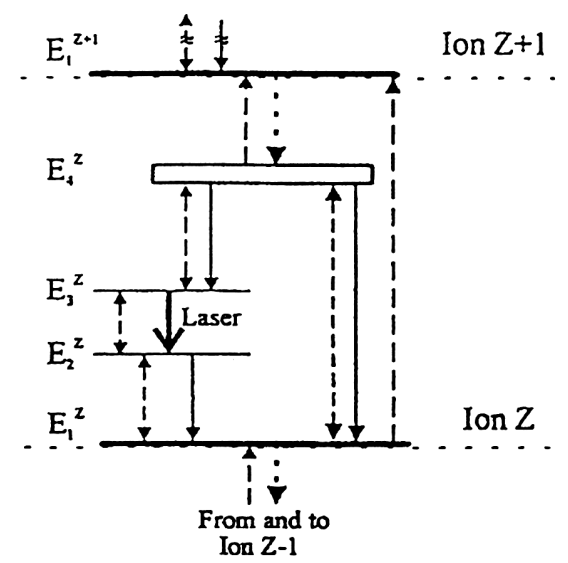

Fig. 4. Simplified laser excitation model energy level scheme. Solid line: radiative decay channels; dotted line: recombination processes; dashed line: collisional excitation-deexcitation and ionization processes.

strengths with the laser transition levels were determined and their parameters included in equation set (1).

Laser levels are mainly populated by electron impact and cascade recombination, and their relative weights differ for different ions. We will restrict our analysis to the optically allowed transitions since their cross sections maintain a large value for a much broader range than the optically forbidden ones [25]. Besides, calculations show that the upper laser level has a stronger transition probability to the lower laser level than to the rest of the levels. The upper level has also a strong connection with higher levels that populate it, with an oscillator strength value of about unity. Based on these facts, a four-level model turns out to be the most favorable scheme to describe the excitation processes that lead to laser action for the different ions.

Fig. 4 shows a simplified diagram of the excitation mechanisms that give rise to inversion population. Contributions of electronic impact, radiative and collisional decay, and recombination cascade processes were taken into account in all cases for the optically allowed transitions. The population is transferred by electron impact from the ground level of each ion $E_{1}^{z}$ to higher excited levels $E_{4}^{z}$. The latter may receive (depending on the configuration) cascade contributions due to recombination processes [18]. $E_{4}^{z}$ and $E_{3}^{z}$ populations are connected by radiative decay and excitation-deexcitation electron collision processes [17]. Since, in all cases, the $E_{3}^{z}$ lifetime is much larger than $E_{2}^{z}$, the population of state 3 exceeds that for state 2 . Based on these assumptions, we will analyze in detail some of the laser transitions belonging to different ions.

Calculations showed that the upper laser level of the 4060.45- $\AA$ laser line belonging to Xe III is populated mainly by dielectronic recombination from the ground Xe IV level: $5 s^{2} 5 p^{3}{ }^{4} S_{3 / 2}$.

The upper level $E_{3}$ of Xe V laser transitions is filled mainly by cascading from the $5 s 5 p^{2} 4 f$ and $5 s^{2} 5 p 7 d$ configurations $\left(E_{4}\right)$, and these are populated by electron impact from the ion ground level in a multistep process.
TABLE II

Calculated and Measured laser Gain

\begin{tabular}{c|c|c|c|c}
\hline \multirow{2}{*}{$\lambda[\AA]^{(a)}$} & \multicolumn{3}{|c|}{ Mcasurcd Gain $[d B / m]$ and } & Calculated Gain \\
\cline { 2 - 4 }$($ air $)$ & Ref. [28] & Ref. [14] & This work & \\
\cline { 2 - 4 }$[d B / m]$ \\
\hline 3246.99 & - & - & $2.8\{70-95\}$ & 3.0 \\
3350.03 & - & - & $2.7\{85-99\}$ & 2.7 \\
3645.48 & - & - & $6.1\{94-100\}$ & 6.5 \\
3803.26 & - & - & $2.5\{93-96\}$ & 2.2 \\
4060.45 & - & - & $2.5\{75-85\}$ & 2.5 \\
4954.13 & $6.3\{88-100\}$ & $7.3\{84-99\}$ & $6.2\{85-99\}$ & 6.1 \\
5007.80 & $2.3\{88-100\}$ & - & $2.6\{85-99\}$ & 2.7 \\
5260.19 & $3.6\{88-100\}$ & $4.0\{85-99\}$ & $2.9\{85-99\}$ & 3.5 \\
5352.92 & $5.2\{88-100\}$ & $6.4\{85-99\}$ & $4.5\{85-99\}$ & 5.0 \\
5394.62 & $3.6\{88-100\}$ & $4.4\{84-99\}$ & $3.6\{85-99\}$ & 3.5 \\
5955.67 & $=$ & $2.7\{75-99\}$ & $2.4\{80-99\}$ & 2.3 \\
\hline
\end{tabular}

(a) Wavelengths were taken from [24].

The upper laser level $E_{3}$ of the Xe VII $\left(5 s 4 f^{1} F_{3}\right)$, is populated both by radiative decay from $E_{4}$ and by recombination from the Xe VIII ground level. Our calculations showed that the latter process is dominant for the $T_{e}$ peak values considered here.

Finally, for Xe VIII, the $E_{3}$ levels are mainly populated by a strong radiative decay from the upper configurations, with transition probabilities of the order of $10^{12} \mathrm{~s}^{-1}$. In turn, these levels are populated mainly by recombination from the $4 d^{10}$ configuration belonging to Xe IX.

\section{LASER GAIN}

The gain coefficient per unit length for different lines appearing in Table II can be calculated using the following relation [26]:

$$
G_{32}=\frac{1}{8 \pi c}\left(\frac{\Delta \lambda}{\lambda}\right)^{-1} A_{32} \lambda^{3} g_{3}\left(\frac{N_{3}}{g_{3}}-\frac{N_{2}}{g_{2}}\right)
$$

where $A_{32}$ is the radiative transition rate, $N_{i}$ and $g_{i}$ are the density population and the statistical weights corresponding to level $i, \Delta \lambda / \lambda$ is the relative linewidth, and $c$ is the speed of light.

The linewidth is given by Doppler broadening and by collisions between electrons and ions. The former can be calculated from [25], and the latter from an expression proposed by Griem [27]. Under our experimental conditions, calculations show that the last term is dominant.

$A_{32}$ values were obtained from RHF calculations. The population densities $N_{3}$ and $N_{2}$ were determined by solving (1) - (5) for typical excitation values [17] and including the main levels that interact with each laser level. The electron temperature $\left(T_{e}\right)$ is increased until laser threshold is reached for each line. The population densities $T_{3}$ and $N_{2}$ obtained under these conditions are introduced in (6).

The small-signal laser gain for the main known transitions was measured in the usual way, introducing a known loss into 


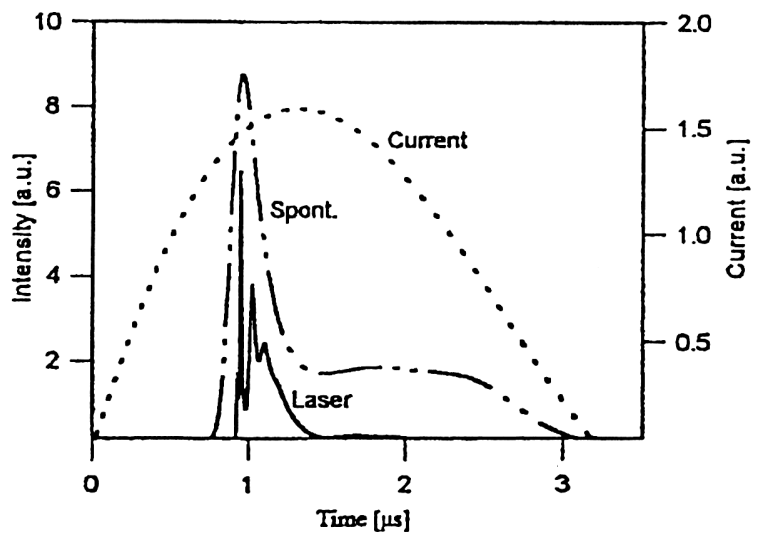

Fig. 5. Calculated temporal distribution of the spontaneous and stimulated emission near threshold for a Xe VIII $(\lambda=3350.03 \AA)$ line.

the cavity until threshold was reached for a particular line. The results can be seen in Table II, together with values from other authors for comparison [14], [28].

Theoretical results are in general agreement with experimental data. For Xe VIII, the spontaneous transition probabilities are lower than those corresponding to other ions, although it is compensated for by the large radiative-collisional decay rate mentioned previously.

\section{TEMPORAL ANALYSIS}

In order to analyze the temporal characteristics of the emission, (1) was solved numerically for typical discharge parameters. All the levels that take place in the excitation-deexcitation processes giving rise to laser action were included. The electron temperature is increased until the shape of the calculated spontaneous emission reproduces the experimental results. After that, the stimulated emission terms (2) and (3) were included in (1), and the whole system was solved together with (4) and (5)

Calculations shows that laser oscillations are dependent on the pumping rate of the levels. As expected, they appear near threshold, in agreement with previous work [16]. This fact was more clearly observed in lines belonging to higher ionization stages such as Xe VII and Xe VIII, since, under our experimental conditions, they operate near threshold. This is theoretically supported by the lower transition probability for the upper laser levels belonging to several of these lines. Near threshold, the calculated emission presents a large number of oscillations. This situation holds, for example, when $A_{32}$ is low, as in the typical case for Xe VIII. Fig. 5 shows the temporal evolution of the spontaneous and stimulated emission for the 3350.03- $\AA$ line of the Xe VIII. The result agrees with that shown in Fig. 1 for the same line.

When the electron temperature is increased from threshold, oscillations disappear and the stimulated emission follows the temporal shape of the spontaneous emission. This agrees with the fact that the lower laser level radiative decay rate is much larger than that for the upper laser level (Table I). Fig. 6 shows the temporal shape of the spontaneous emission for a Xe VII transition and the $3645.48-\AA$ stimulated emission, reproducing

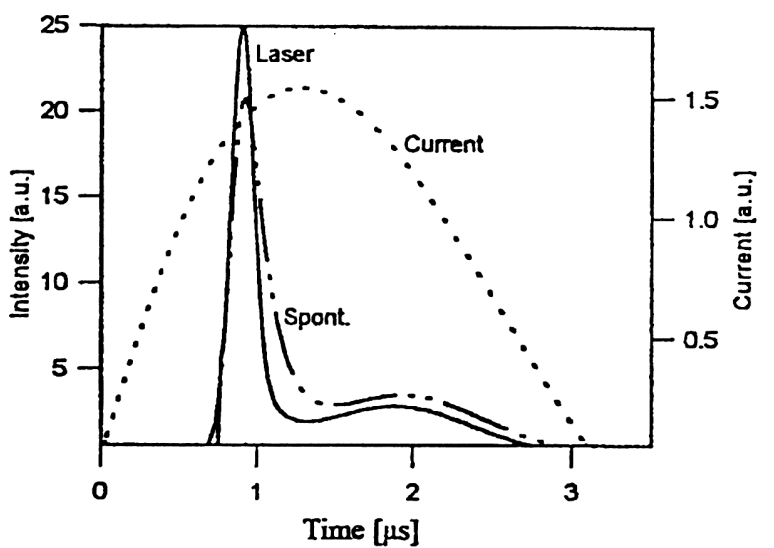

Fig. 6. Calculated output of the spontaneous and stimulated emission for the $\lambda \equiv 3645.48-\AA$ line belonging to Xe VII.

the experimental observation of Fig. 2. Further increases of the electron temperature produce an intense single laser pulse, as was shown in previous works [10], [16].

\section{CONCLUSIONS}

The temporal evolution of more than $20 \mathrm{UV}$-visible xenon laser lines and their corresponding spontaneous emission from a multi-ionic low-pressure plasma is analyzed using timeresolved spectroscopy. RHF calculations on Xe III to Xe IX were carried out to determine spontaneous transition probabilities and lifetimes of relevant levels involved in the laser emission. Results show that the upper laser spontaneous transition rate to the lower laser level is larger than to the rest of the levels. Moreover, the upper level lifetime is one or two orders of magnitude greater than that for the lower state. Using a collisional-radiative model based on electron impact and cascade processes, we studied the dynamics of the plasma. The numerical solution of this model, including the stimulated terms, predict the observed oscillations near threshold which agrees with experimental results. It can also be used to determine the optimum lasing conditions for each line. The results also indicated that, under certain experimental conditions, the strong $3645.48-\AA$ line can also be made to lase in an extended mode, giving rise to a quasi-CW temporal profile, similar to the visible lines reported in [29]. We found in most of the laser lines that both the electron impact and recombination mechanisms are responsible for population inversion. The agreement between experimental small-signal measured gain and the calculated gain values supports the proposed model. The model is being applied to other multiionic rare-gas laser systems.

\section{REFERENCES}

[1] W. B. Bridges and A. N. Chester, "Visible and UV laser oscillations at 118 wavelengths in ionized neon, argon, krypton, xenon, oxygen, and other gases," Appl. Opt., vol. 4, pp. 573-580, 1965.

[2] " "Spectroscopy of ion lasers," IEEE J. Quantum Electron., vol. $\overline{\mathrm{QE}-1}$, pp. 66-84, 1965.

[3] P. K. Cheo and H. G. Cooper, "Ultraviolet ion laser transitions between 2300 and 4000 A," J. Appl. Phys., vol. 36, pp. 1862-1865, 1965. 
[4] E. Gallego Lluesma, A. Tagliaferri, C. Massone, M. Garavaglia, and M. Gallardo, "Ionic assignment of unidentified of xenon laser lines," J. Opt. Soc. Amer., vol. 63, pp. 362-364, 1973.

[5] T. W. Hänsch, A. L. Schawlow, and P. Toschek, "Simple dye laser repetitively pumped by a xenon ion laser," IEEE J. Quantum Electron., vol. QE-9, pp. 553-554, 1973.

[6] D. W. Fahey and L. D. Schearer, "A xenon ion pumped blue dye laser," IEEE J. Quantum Electron., vol. QE-14, pp. 220-221, 1978.

[7] L. D. Schearer, "A high-power pulsed xenon ion laser as a pump source for a tunable dye laser," IEEE J. Quantum Electron., vol. QE-11, pp. 935-937, 1975.

[8] J. Pinard and L. Liberman, "A frequency locked, single mode pulsed dye laser. Application to single frequency tunable UV generation," Opt. Commun., vol. 20, pp. 344-346, 1977.

[9] J. Tocho, R. Duchowicz, M. Gallardo, and M. Garavaglia, "Frequency locking in double-pumped dye laser," IEEE J. Quantum Electron., vol. QE-17, pp. 1591-1592, 1981.

[10] R. Duchowicz, D. Schinca, and M. Gallardo, "New analysis for the assignment of UV-visible ionic Xe laser lines," IEEE J. Quantum Electron., vol. 30, pp. 155-159, 1994.

[11] M. Gallardo, M. Raineri, J. Reyna Almandos, H. Sobral, and F. Callegari, "Revised and extended analysis in four times ionized xenon Xe V," J. Quantitative and Radiative Transfer. vol. 61, pp. 319-327, 1998.

[12] M. Larsson, A. Gonzalez, R. Hallin, F. Heijkenskjōld, R. Hutton, A. Langereis, B. Nynström, B. Sullivan, and A. Wönnström, "Wavelengths and energy levels of Xe VII and Xe VIII obtained by collision-based spectroscopy," Phys. Scr., vol. 51, pp. 69-75, 1995.

[13] M. Wang, A. Amesen, R. Hallin, F. Heijkenskjöld, A. Langereis, M. Larsson, $C$. Nordling, and A. Wönnström, "Collision-based spectroscopy of Xe VIII Rydberg states," J. Opt. Soc. Amer. B, vol. 13, pp. 1650-1658, 1996.

[14] A. Papayoanou, R. Buser, and I. Gumeiner, "Parameters in a dynamically compressed xenon plasma laser," IEEE J. Quantum Electron., vol. QE-9, pp. 580-585, 1973.

[15] W. Sasaki and S. Saito, "Bouble-pulsed output with a Xe IV laser excited by long current pulse discharges," Appl. Opt., vol. 19, pp. 5-6, 1980.

[16] H. Sobral, D. Schinca, R. Duchowicz, and M. Gallardo, "Gain switching and oscillations in a ionic pulsed xenon laser," Pure Appl. Opt., vol. 5 , pp. 1011-1019, 1996.

[17] H. Sobral, D. Schinca, M. Gallardo, and R. Duchowicz, "Time dependent study of a multi-ionic xenon plasma," J. Appl. Phys., vol. 85, pp. 69-73, 1999.

[18] I. Sobelman, L. Vainshtein, and E. Yukov, Excitations of Atoms and Broadening of Spectral Lines. Berlin, Germany: Springer-Verlag, 1981.

[19] A. Siegman, Lasers. Mill Valley, CA: University Sci., 1986.

[20] R. Cowan, The Theory of Atomic Structure and Spectra. Berkeley, CA: UC Berkeley, 1981.
[21] W. Persson, C. Wahlström, G. Bertuccelli, H. Di Rocco, J. Reyna Almandos, and M. Gallardo, "Spectrum of doubly ionized xenon (Xe III), Phys. Scr., vol. 38, pp. 347-369, 1988

[22] J. Marling, "Ultraviolet ion laser performance and spectroscopy-Part I: New strong noble-gas transitions below $2500 \AA$," IEEE J. Quantum Electron., vol. QE-11, pp. 822-834, 1975.

[23] A. Tauheed, Y. Joshi, V. Kaufman, J. Sugar, and E. Pinnington, "Analysis of the $5 s^{2} 5 p^{2}-\left(5 s 5 p^{3}+5 s^{2} 5 p 5 d+5 s^{2} 5 p 6 s\right)$ transitions of four-times ionized xenon (Xe V)," J. Opt. Soc. Amer. B, vol. 10, pp. 561-565, 1993.

[24] M. Gallardo and J. Reyna Almandos, private communication.

[25] C. Willett, Introduction to Gas Lasers: Population Inversion Mechanisms. Oxford, U.K.: Pergamon, 1974.

[26] M. Pöckl, M. Hebenstreit, T. Neger, and F. Aumayr, "Time-dependent collisional-radiative model for capillary discharge plasmas," J. Appl. Phys., vol. 76, pp. 733-737, 1994.

[27] H. Griem, "Semiempirical formulas for the electron-impact widths and shifts of isolated ion lines in plasmas," Phys. Rev., vol. 165, pp. 258-266, 1968.

[28] W. Simmons and R. Witte, "High-power pulsed xenon ion lasers," IEEE J. Quantum Electron., vol. QE-6, pp. 466-469, 1970.

[29] W. Bridges and G. Mercer, "CW operation of high ionization states in a xenon laser," IEEE J. Quantum Electron., vol. QE-5, pp. 476-477, 1969.

H. Sobral, photograph and biography not available at the time of publication.

M. Raineri, photograph and biography not available at the time of publication.

D. Schinca, photograph and biography not available at the time of publication.

M. Gallardo, photograph and biography not available at the time of publication.

R. Duchowicz, photograph and biography not available at the time of publication. 